\title{
Simulation of Magnetophoretic Separation Processes in Dispersions of Superparamagnetic Nanoparticles in the Noncooperative Regime
}

\author{
Jordi S. Andreu, ${ }^{1,2}$ Pablo Barbero, ${ }^{2}$ Juan Camacho, ${ }^{2}$ and Jordi Faraudo ${ }^{1}$ \\ ${ }^{1}$ Materials Simulation and Theory Department, Institut de Ciència de Materials de Barcelona (ICMAB-CSIC), \\ Campus UAB, 08193 Bellaterra, Spain \\ ${ }^{2}$ Departament de Física, Universitat Autònoma de Barcelona, Campus UAB, 08193 Bellaterra, Spain
}

Correspondence should be addressed to Jordi S. Andreu, jandreu@icmab.es

Received 15 December 2011; Accepted 30 January 2012

Academic Editor: Carlos Martinez-Boubeta

Copyright (C) 2012 Jordi S. Andreu et al. This is an open access article distributed under the Creative Commons Attribution License, which permits unrestricted use, distribution, and reproduction in any medium, provided the original work is properly cited.

\begin{abstract}
Magnetic separation has gained much attention due to its implications in different fields, becoming feasible as an alternative to existent technologies at the industrial and lab scale. Substantial efforts are focused to improve the magnetic particles used in these applications. Here we show how a relatively simple and low-cost simulation strategy (tracer simulations) can be employed to predict the effect of various key factors in magnetic separation processes, namely, particle properties and magnetic separator designs. For concreteness, we consider here specific problems in magnetic separation. The first one is the effect of different profiles of the magnetic field in the separation of magnetic nanoparticles, and the second one is the magnetophoresis of colloidal particles in a dispersion of magnetic nanoparticles.
\end{abstract}

\section{Introduction}

The manipulation of magnetic particles by the use of inhomogeneous magnetic fields has emerged as a topic of great interest in a wide range of research and technological areas [1]: from wastewater treatments $[2,3]$ or pollutants removal [4] to biomedical applications like protein isolation, drug delivery, magnetic hyperthermia, or magnetic particle imaging $[5,6]$. The use of inhomogeneous magnetic fields to drive magnetic particles apart from solution, what is known as magnetic separation or magnetophoresis, has provided new techniques capable to improve standard technologies, especially in biotechnological applications [7].

The idea behind magnetic separation is to take advantage of the distinctive magnetic response of the particles in solution to remove them from complex mixtures by the use of applied inhomogeneous magnetic fields [8]. In a wide range of applications, magnetic particles are typically functionalized with proper chemical groups, designed to bind to specific nonmagnetic components, thus enabling the separation of nonmagnetic materials by combining the use of magnetic particles and magnetic fields. This combination has many advantages over traditional fixed-bed separation methods, such as activated carbon adsorption for organics and affinity chromatography for proteins. In particular, magnetic nanoparticles offer large exposed surface areas without the use of porous materials, which are often plagued by high mass transfer resistances [9]. Therefore, it is not surprising that magnetic separation has been presented as an alternative to typical centrifugation and filtration steps in industrial processes as well as in lab applications. In the biomedical field, magnetic separation may help to overcome some disadvantages of standard column liquid chromatography in the separation of proteins and peptides, and it serves as a basis of various immunoassays systems. Moreover, magnetic separation can also be used to concentrate large volumes of diluted protein solutions in a very gentle way [7].

The basic ingredients in any magnetic separation application are two: the selection of appropriate magnetic particles 
and the design of the magnetic separator. Typically, the particles employed in these applications are superparamagnetic particles. One has to bear in mind that superparamagnetism emerges as a quantum effect in some ferromagnetic and ferrimagnetic materials, below the single domain size. This implies that this phenomenon is limited to nanocrystals of size below a certain critical size which depends on the material [10]. Since the magnetic force is proportional to the particle magnetization, superparamagnetic nanoparticles with large magnetic response are desired. A standard way to enhance the magnetic response of the carrier particles is to synthesize larger particles by embedding superparamagnetic nanocrystals in a matrix of nonmagnetic material (such as polystyrene [11] or silica [12]), thus preserving the superparamagnetic behavior of these crystals and guaranteeing the stability and biocompatibility of the solutions. In this manner, particles with larger magnetizations are obtained, increasing the magnetic response under an external field. Nevertheless, one has to balance this increase on the magnetic response (which could enhance the separation process) with the reduction on the active surface area of the particles, implying a reduction on the capture and retention of target entities. Actually, a wide range of different particles are already commercially available (from nanoparticles to larger superparamagnetic colloids) combining different magnetic response, size and decorated surfaces designed to target specific components.

A second issue about the separation process is the application of a specific magnetic field over the target sample, inducing a magnetic moment in the carrier particles. This magnetic field has not only to induce a magnetic moment but also to generate a magnetic gradient (which produces a magnetic force on the particle) in order to drive carrier particles apart from solution. Then, the conditions to be fulfilled by the magnetic field source are two: it has to induce large magnetizations but also a gradient in the intensity of the magnetic field. The simplest option to induce magnetophoresis in a lab tube or vial is by the application of a simple bar magnet, but this option is highly inefficient, since typically only those particles near the magnet really experience enough magnetic force to move. However, it is possible to obtain efficient magnetic separators by combining permanent magnets in convenient arrangements in order to generate magnetic fields suitable for magnetic separation. Among the possible arrangements of the magnets, we will discuss here the advantages and drawbacks of two possible cylindrical tube geometries, which have been called open and closed arrangements. Essentially, the closed structure consists of an arrangement of magnets around the tube containing the suspension, which generates a uniform magnetic gradient pointing towards the wall of the tube (see, e.g., [13-17]). In this case, one obtains uniform magnetophoretic conditions, a desirable feature in order to characterize, mod$\mathrm{el}$, and scale-up the magnetophoresis process. The open-type magnetic separator $[18,19]$ is similar to the previous one, but in this case the design contains an aperture, that is, there is a region near the tube walls which does not contain magnets. These open structures are designed to operate directly to test tube racks and helps on the visual monitoring of the process. In a preliminary communication [20], we noted that these two different separator designs induce substantial differences in the dynamics of the magnetic separation process.

Here, we propose a simple simulation methodology which allows to model the magnetophoretic separation of nanoparticles inside different designs of magnetic separators. As a first application, the methodology proposed here will be employed to compare the performance of open and closed separator designs. In a second one, we will study the magnetophoretic separation process of a mixture containing particles with different sizes and magnetic responses.

\section{Tracer Simulation of Magnetophoresis of Superparamagnetic Nanoparticles}

2.1. Basic Equations and Simulation Methodology. Let us start by describing the equations of motion of superparamagnetic nanoparticles (NPs) in a liquid dispersion under the effects of an external magnetic field. In this situation, NPs will move in the direction of the magnetic gradient (magnetophoresis). As shown experimentally and theoretically in previous works $[13,17]$, we have two different kinds of magnetophoretic separation processes. The first case is called cooperative magnetophoresis, and it is characterized by fast separation times (magnetophoretic velocities can be roughly estimated as of the order of a cm per minute) which depend strongly on the concentration of the sample. It is usually found for composite colloids of several hundreds of $\mathrm{nm}$ in diameter (made of magnetic NPs embedded in a nonmagnetic matrix) and its driving force is the reversible formation of chains of colloids under the magnetic field [13]. The second case corresponds to noncooperative magnetophoresis, and it is typically found for dispersions of small NPs [17]. In this case, magnetophoretic velocities are much lower, and they do not depend on particle concentration; instead, they depend strongly on the magnetic gradient and the design of the magnetic separator $[17,20]$. In this case, the magnetophoretic separation process is ruled by the individual motion of the superparamagnetic NPs. In addition to experimental characterization of both situations, we have formulated a mathematical criterion which allows us to predict whether we will observe cooperative or noncooperative magnetophoresis. This criterion (see [17] for details) establishes that cooperative magnetophoresis is observed when the aggregation parameter $N^{*}$ verifies $N^{*}>1$. This parameter is given by

$$
N^{*}=\sqrt{\phi_{0} e^{\Gamma-1}},
$$

where $\phi_{0}$ is the initial volume fraction of particles in suspension, and $\Gamma$ is the magnetic coupling parameter characterizing the strength of magnetic interaction of particles at contact as compared with thermal agitation and defined as

$$
\Gamma=\frac{\mu_{0} m_{s}^{2}}{2 \pi d^{3} k_{B} T} .
$$

In (2) $\mu_{0}$ is the permeability of the free space, $m_{s}$ is the magnetic moment of a particle at saturation, $d$ is its diameter, and $T$ the absolute temperature.

In this work, we will consider only the noncooperative case characterized by $N^{*}<1$, which is typically the case for 
dispersions of NPs. For example, a $10 \mathrm{~g} / \mathrm{L}$ dispersion of superparamagnetic $\gamma$ - $\mathrm{Fe}_{2} \mathrm{O}_{3}$ NPs (diameter $12 \mathrm{~nm}$ ) employed in [17] provide $\Gamma=2.5$ and $N^{*}=0.1$.

Under these conditions, we thus need to consider only the individual motion of NPs in the magnetic gradient to obtain the magnetophoretic behavior, ignoring the interaction between NPs. The magnetophoretic velocity of a NP immersed in a fluid with viscosity $\eta$ submitted to a magnetic gradient can be obtained as follows. The magnetic force acting on a magnetic particle can be written as

$$
\vec{F}_{\mathrm{mag}}=\mu_{0} V \rho_{p}(\vec{M}(H) \cdot \nabla) \vec{H},
$$

where $\vec{H}$ is the magnetic field, $V$ is the volume of the particle, $\rho_{p}$ is its density, and $\vec{M}$ is the magnetization of the particle per unit mass. Notice here that this expression assumes that the magnetization of the particle is uniform [8] so that the magnetic moment is given by

$$
m(H)=V \rho_{p} M(H) .
$$

Nevertheless, in the case of a superparamagnetic particle, its total magnetic moment aligns parallel to the applied magnetic field and (3) can be written as

$$
\vec{F}_{\mathrm{mag}}=\mu_{0} V \rho_{p} M(H) \vec{\nabla} H,
$$

where $H$ is the modulus of the magnetic field. On the other hand, the viscous drag force exerted by the solvent over a single spherical particle of radius $R$ is

$$
\vec{F}_{\text {vis }}=-6 \pi \eta R \vec{v}
$$

The magnetophoretic velocity of a particle in the steady state is obtained by balancing the magnetic force $F_{\text {mag }}$ and the viscous drag force $F_{\mathrm{vis}}$ exerted by the solvent:

$$
\vec{v}=\frac{2 \mu_{0} \rho_{p} M(H) R^{2}}{9 \eta} \vec{\nabla} H .
$$

In order to apply (7) in a real situation, one needs to know not only the profile of the applied magnetic field but also a full characterization of the magnetic response $M(H)$ of the NPs. In our calculations, we will assume that the magnetization $M(H)$ of a single superparamagnetic nanoparticle under an external magnetic field $H$ is described within a good approximation by a Langevin function typical in theoretical descriptions of this superparamagnetic behavior [10, 21-23]

$$
M(H)=M_{s} \mathcal{L}\left[b \mu_{0} H\right], \quad \mathcal{L}[x]=\operatorname{coth} x-\frac{1}{x},
$$

where $M_{s}$ denotes the saturation magnetic moment per unit mass, and $b$ is related to $M_{s}$ and $R$ by (see $[17,22]$ )

$$
b=\frac{m_{s}}{k_{B} T}=\frac{4 \pi R^{3} M_{s} \rho_{p}}{3 k_{B} T} .
$$

The value of $b^{-1}$ can be interpreted as a characteristic magnetic field required to reach saturation. Equations (7)(9) allow one to predict the magnetophoretic motion of a superparamagnetic NP if the spatial profile of the magnetic field of the magnetic separator is known.

In the case of a very simple geometry for the magnetic field, it has been possible [17] to obtain analytically an exact equation for the trajectory of a NP inside a magnetic separator and also obtain the kinetics of the separation process (number of particles remaining in solution as a function of time). However, for a general magnetic field geometry, finding an analytical solution is not possible. The option explored in this paper will be to perform simulations of tracer particles under known magnetic profiles. In this simulation method, each particle tracer is not intended to represent a real particle but it effectively describes the dynamics of a given particle under the external conditions imposed (tracer simulations are common in fields such as fluid mechanics, see, e.g., [24]).

The simulation technique is as follows. First of all, we need to know the geometry of the magnetic separator and the magnetic field $H(\vec{r})$ in all points inside the magnetic separator. Then, we consider the motion of $N_{p}$ tracers inside the given geometry and magnetic field. Initially $(t=0)$, the positions $\vec{r}_{i}(t=0)\left(i=1, \ldots, N_{p}\right)$ of these tracers are generated to be distributed uniformly inside the magnetic separator. The simulation then proceeds by assuming that each of these tracers behaves as a superparamagnetic NP. The position $\vec{r}_{i}(t)$ of a tracer evolves obeying

$$
\frac{d \vec{r}_{i}}{d t}=\vec{v}\left(\vec{r}_{i}\right)
$$

where the magnetophoretic velocity $\vec{v}\left(\vec{r}_{i}\right)$ is computed from (7)-(9) taking into account the local value of the magnetic field and the magnetic gradient evaluated at position $\vec{r}_{i}$. The equation of motion of the tracers ((10) supplemented with (7)-(9)) is integrated numerically in discrete time steps $\Delta t$ by employing a Verlet [25] type integration algorithm which provides good accuracy at a reasonable cost of CPU time. All these calculations were implemented in a $\mathrm{C}$ code developed in house, which is available under request to the authors.

2.2. Validation of the Simulation Methodology. In this subsection, we will consider a magnetophoretic separation problem for which we obtained both experimental results and an analytical solution. Comparison of the results of our simulations with previously known results is a necessary step in order to ensure the validity of our simulation approach. After this validation step, we will employ our simulation method in the following subsection to explore other situations in which previous theoretical results are not available.

Here we consider the closed geometry for the magnetic separator, similar to the actual separators employed in recent experimental works $[13,15-17]$. The geometry of the separator is a cylinder (radius $L=1.5 \mathrm{~cm}$ ), and it contains a magnetic field increasing from zero in the center to a maximum value at the walls, as shown in Figure 1 (see also inset in Figure 2 for a sketch of the separator). Note that the gradient of the modulus of the magnetic field is approximately uniform inside the system $(\approx 30 \mathrm{~T} / \mathrm{m})$. The parameters for the simulation were selected in order to match the experimental system considered in Figure 1 of [17]. In 


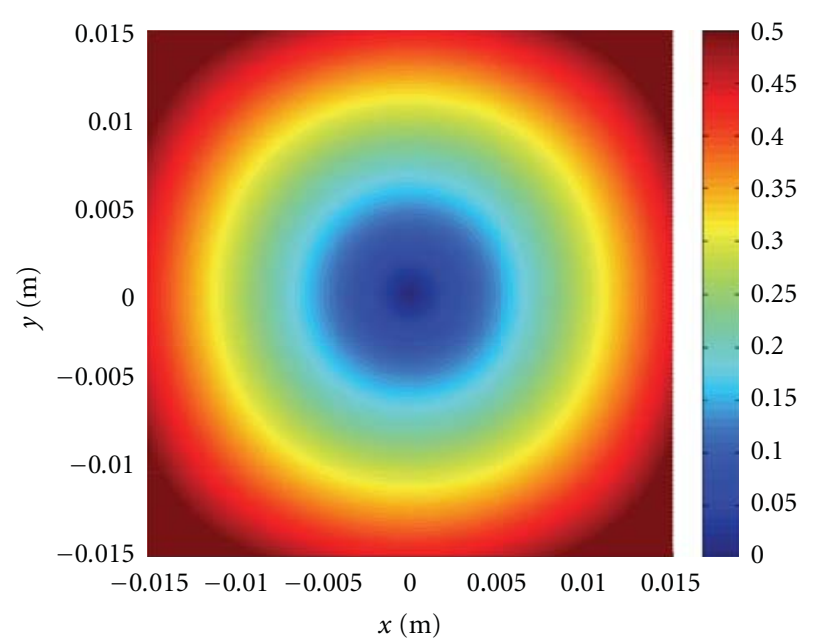

(a)

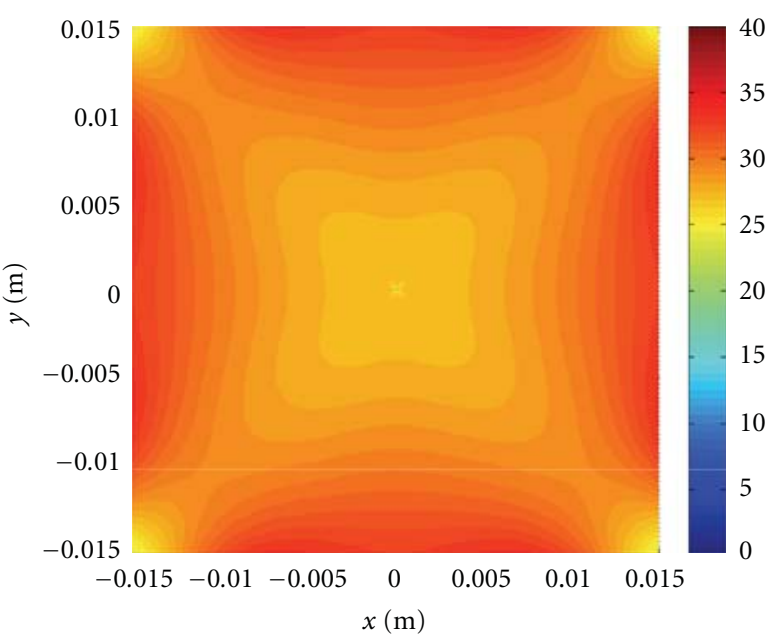

(b)

Figure 1: Profile of the magnitude (modulus) of the quadrupolar magnetic field in Tesla (a) and its gradient in Tesla/m (b) for the closed type magnetic separator (top view) employed in the simulations. Note that the magnitude of gradient of the field intensity corresponds to $\approx 30 \mathrm{~T} / \mathrm{m}$ in most regions of the separator; however, inhomogeneities due to the quadrupolar nature of the field can be clearly seen.

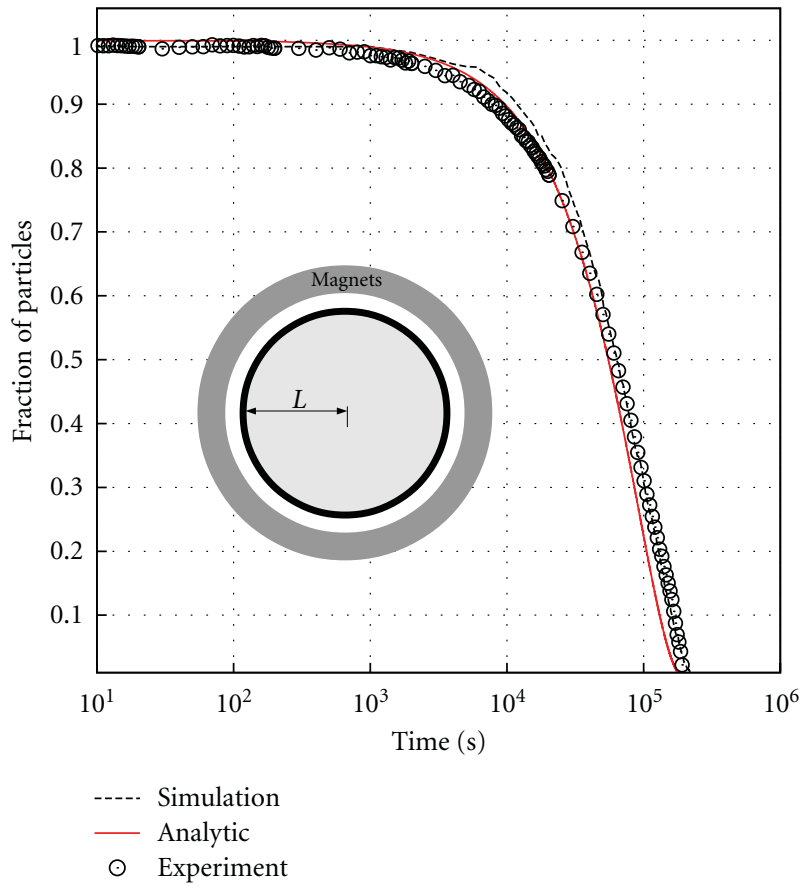

Figure 2: Magnetophoretic separation of superparamagnetic $\gamma$ $\mathrm{Fe}_{2} \mathrm{O}_{3}$ NPs of diameter $12 \mathrm{~nm}$ inside a $30 \mathrm{~T} / \mathrm{m}$ magnetic separator. Comparison between the simulations performed here, the analytical solution and the experimental results reported in [17] for the evolution of the fraction of particles remaining in the separator. Inset: sketch (top view) of the magnetic separator.

this experiment, a $10 \mathrm{~g} / \mathrm{L}$ dispersion of superparamagnetic $\gamma$ $\mathrm{Fe}_{2} \mathrm{O}_{3} \mathrm{NPs}$ (diameter $12 \mathrm{~nm}$ ) was placed inside the separator. The magnetization curve for these NPs was given also in [17], and it was shown that they obey (8) and (9) with $m_{s}=(4 / 3) \pi R^{3} \rho_{p} M_{s}=3 \times 10^{-19} \mathrm{~J} / \mathrm{T}$ and $b=68 \mathrm{~T}^{-1}$. The employed solvent was water (with viscosity $\eta \simeq 0.001 \mathrm{~Pa} \cdot \mathrm{s}$ at $298 \mathrm{~K})$. Our tracer simulations were performed considering $N_{p}=10^{3}$ tracer particles and a time step of $\Delta t=10^{2} \mathrm{~s}$. The simulations were performed until a simulation time of $2 \times$ $10^{5} \mathrm{~s}$. The calculations required only $26 \mathrm{~min}$ of CPU running in a single core of an AMD Opteron Magny Cours 6136 processor. During the simulation, we saved the trajectories of the tracer particles for further analysis. From these results, we estimated the concentration profile of the NPs at different times and we also computed the time evolution of the fraction of particles inside the dispersion (i.e., the number of tracers which have not reached the walls of the system divided by the total number of tracers). This last quantity is compared in Figure 2 with the experimental results obtained in [17]. We also show the results corresponding to the analytical expression developed in [17]. Our simulation results are in good agreement with these previous results, thereby validating our simulation technique.

2.3. Comparison between Different Separator Designs. Now, we employ our simulation methodology to compare the performance of two different designs of magnetic separators. The first design we consider here is the one considered in the previous subsection, which we will call "closed type" separator from now on. As we said previously, the main advantage of this geometry for the magnetic separator is the fact that the magnetic gradient is approximately uniform inside the system. The second design we will consider here is an "open type" separator. In this case, the geometry of the separator is the same as the closed type considered in the previous subsection, but now part of the magnets were removed. As we have mentioned in the introduction, this partial removal of magnets is made in commercial separators in order to facilitate visual contact with the dispersion during the separation process so that the separation can be monitored easily 


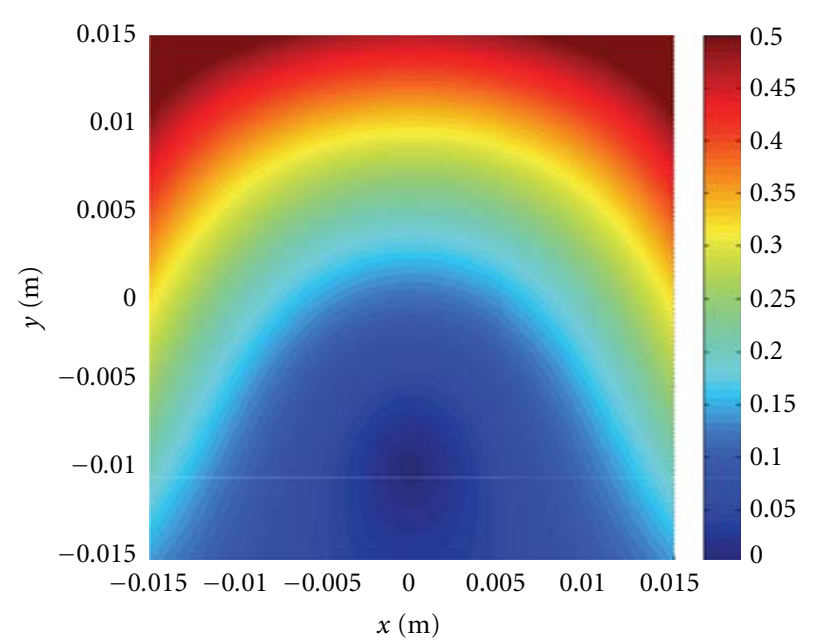

(a)

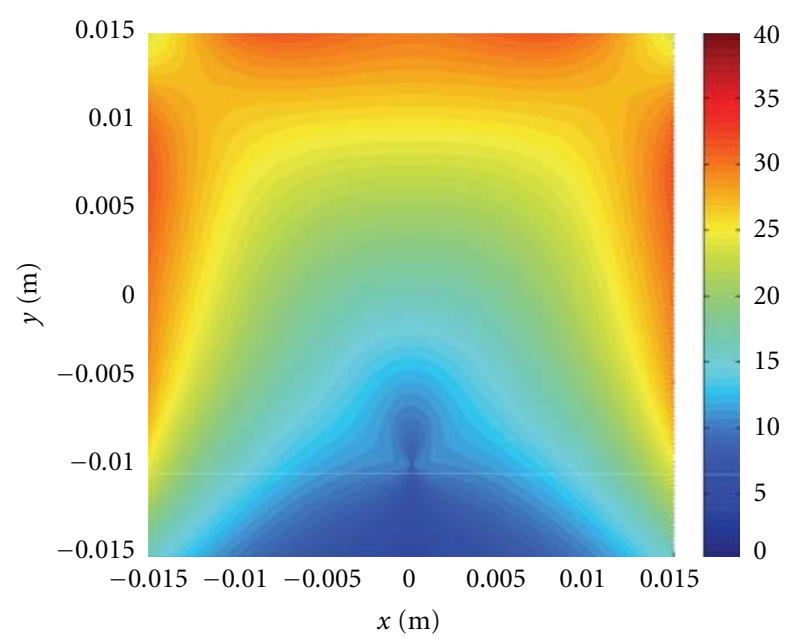

(b)

Figure 3: Profile of the magnitude of the magnetic field $B$ in Tesla (a) and its gradient in Tesla/m (b) in the open-type magnetic separator employed in the simulations.

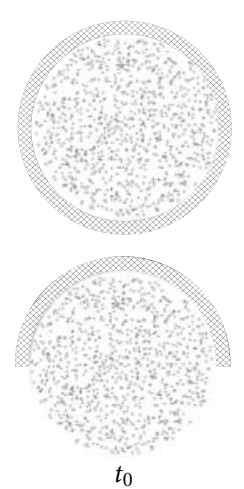

$t_{0}$

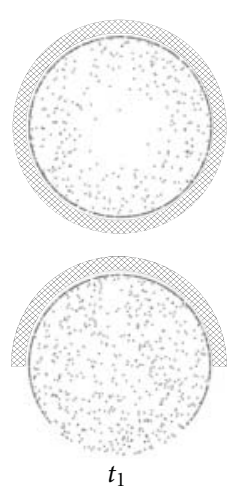

$t_{1}$
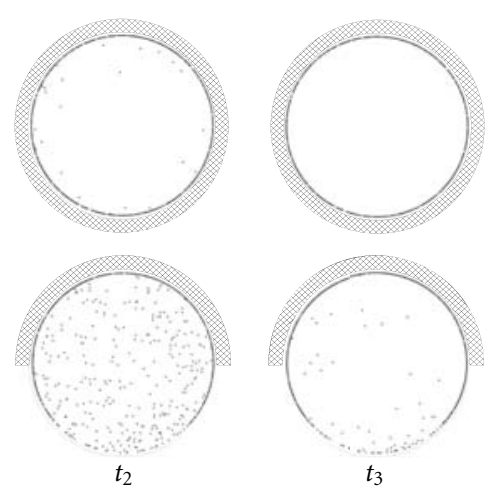

$t_{3}$

FIGURE 4: Series of snapshots extracted from simulations comparing the time evolution of the separation process in the closed-geometry (upper row) and open-geometry (bottom row) schemes. The snapshots are taken from a top view of the cylindrical separator with radial geometry. The different snapshots correspond to different times during separation $\left(t_{0}=0, t_{1}=1 \times 10^{5} \mathrm{~s}, t_{2}=2 \times 10^{5} \mathrm{~s}\right.$ and $\left.t_{3}=5 \times 10^{5} \mathrm{~s}\right)$.

by eye inspection [19] (in closed type separators as the one considered in the previous subsection, monitoring of the separator process is made by an optical sensor, see, e.g., [13]). In Figure 3 we show the profile of the magnetic field generated by a hypothetical open-type magnetic separator constructed by removing half of the magnets from the closed type magnetic separator employed in the previous subsection. In this open case, the magnetic gradient is far from uniform. It is again about $30 \mathrm{~T} / \mathrm{m}$ near the magnets, but now it is less than $10 \mathrm{~T} / \mathrm{m}$ in a substantial part of the separator (the region far from the magnets). We will employ these profiles of magnetic field and gradient in order to compare the performance of the open and closed separator designs in a simulation of a specific example of magnetic separation.

In order to compare the different performance between the open and the closed type separators, we consider the same suspension of $\gamma-\mathrm{Fe}_{2} \mathrm{O}_{3}$ NPs of diameter $12 \mathrm{~nm}$ described in the previous subsection. Now we perform simulations for this suspension in the case of open-type geometry of the magnetic separator. The technical details (number of tracer particles, time step, etc.) were the same as employed in the simulation of the previous subsection. Here, the simulations were performed until a simulation time of $1.2 \times 10^{6} \mathrm{~s}$, and the calculations required $39 \mathrm{~min}$ of CPU in a AMD Opteron Magny Cours 6136 processor. The corresponding results are presented in Figure 4 (snapshots) and Figure 5 (fraction of remaining particles as a function of time). The differences between results for both types of separators are clear. In the closed type geometry, the motion of particles is much more uniform since the magnetic gradient is nearly uniform in the whole system. In this case, separation proceeds by a radial motion of the particles towards the wall (following the magnetic gradient, see (7)), leaving a circle of "clear" solution (free of particles) which increases with time. In the open-type case, the distribution of particles becomes inhomogeneous as separation proceeds, because of the inhomogeneities in the magnetic gradient (see Figure 3). Particles close to the magnets move faster than the ones placed far from the magnets (Figure 4, top) since they experience larger magnetizations and larger magnetic gradients (see Figure 3). Also, 


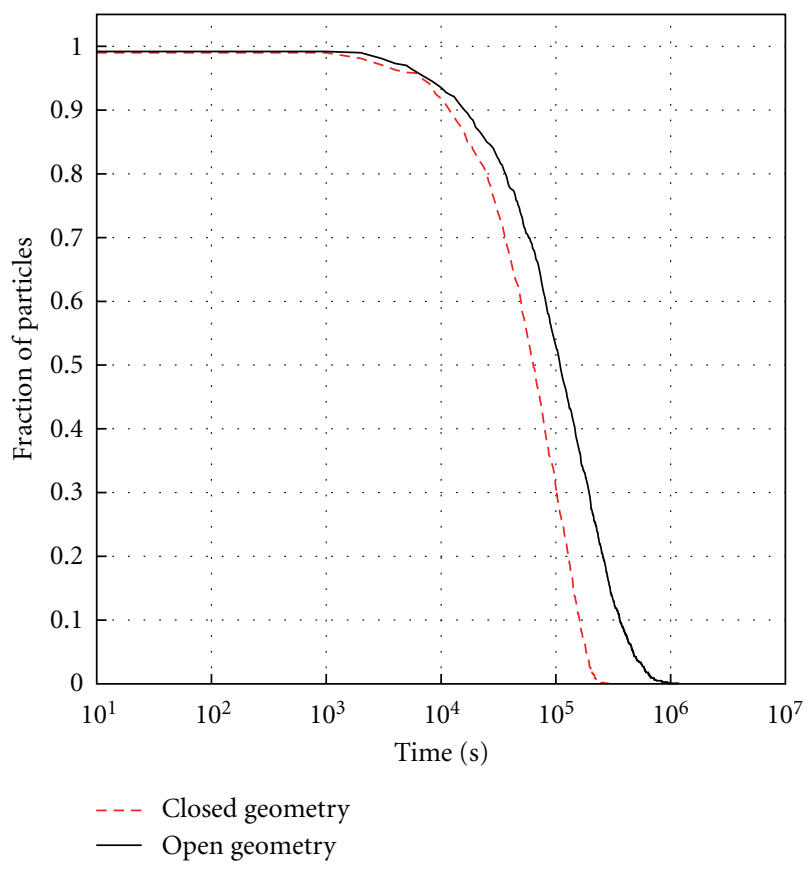

Figure 5: Comparison of the fraction of particles in solution as a function of time as obtained in tracer simulations for open and closed type magnetic separators with magnetic profiles shown in Figures 1 and 3 (see text for details).

these slow-moving particles have to travel distances larger than the cylinder radius in order to reach the walls of the system and become separated from the liquid. As a consequence, separation times are substantially longer in opentype separators than in closed type separators, as shown in Figure 5.

\section{Magnetophoresis of Colloidal Particles in a Magnetic Fluid}

3.1. Motivation and Basic Equations. The problem we would like to consider in this section is the motion of colloidal particles (with sizes of the order of hundreds of nm or larger) in a dispersion containing superparamagnetic NPs. This particularly asymmetric mixture has a fascinating behavior which has received significant attention in recent years. For example, it is possible to induce the assembly and transport of nonmagnetic colloids immersed in a dispersion of superparamagnetic NPs by applying external magnetic fields [26, 27]. Physically, this interesting behavior is due to the fact that a nonmagnetic colloidal particle, immersed in a suspension of NPs, behaves as a magnetic hole with an effective dipole pointing in a direction opposed to that of the local magnetization of the NPs. Hence, after the application of a magnetic field, a nonmagnetic colloid immersed in a dispersion of NPs behaves as an effective super-diamagnetic particle. This effect is not only found in nonmagnetic colloids but also it is possible in the case of composite colloidal particles made of superparamagnetic NPs embedded in a nonmagnetic matrix (e.g., polymer or silica). As demonstrated in $[8,28,29]$ theoretically and experimentally, a colloidal particle in a dispersion of superparamagnetic NPs under a magnetic field $H$ behaves as having an effective magnetic dipole given by

$$
m_{\mathrm{eff}}(H)=m_{c}(H)-\frac{4}{3} \pi R_{c}^{3} n_{p} m_{p}(H),
$$

where $m_{c}(H)$ is the intrinsic magnetic dipole of the colloid (the one observed when the colloid is not embedded in a dispersion of NPs), $R_{c}$ is the radius of the colloid, $n_{p}$ is the local concentration of NPs (in number of particles per unit volume), and $m_{p}(H)$ is the magnetic dipole of the NPs induced by the external field $H$. Equation (11) can be interpreted as a magnetic buoyancy effect due to the different magnetic response of the colloid and its surroundings, as discussed in [8]. Note that a nonmagnetic colloid $\left(m_{c}=0\right)$ will have always $m_{\text {eff }}<0$, that is, a super-diamagnetic behavior with an effective dipole opposite to that of the superparamagnetic NPs. In the case of $m_{c}>0$, the behavior of the colloids can be tuned to an effective superparamagnetic $\left(m_{\mathrm{eff}}>0\right)$ or super-diamagnetic $\left(m_{\mathrm{eff}}<0\right)$ behavior depending on the concentration of NPs.

Here, our interest will be the study of the behavior of a mixture of colloidal particles and NPs in a magnetic separator. Experimentally, this system has been studied in [30] in the case of a closed type separator. The magnetophoretic velocity of the superparamagnetic NPs will obey the same equations discussed in Section 2. In particular, the NPs will move in the direction of the magnetic gradient with a velocity given by (7). The magnetophoretic velocity of a colloidal particle $v_{c}$ can be easily obtained using the same relations derived in Section 2 but taking into account that the magnetic dipole of the colloid is described by $m_{\text {eff }}$ as given by (11). The result is given by

$$
\vec{v}_{c}(\vec{r})=\vec{v}_{p}(\vec{r}) \frac{R_{p}}{R_{c}}\left[\frac{m_{c}}{m_{p}}-\frac{4}{3} \pi R_{c}^{3} n_{p}(\vec{r}, t)\right],
$$

where $\vec{v}_{p}(\vec{r})$ is the magnetophoretic velocity of a NP located at $\vec{r}$, and $n_{p}(\vec{r}, t)$ is the local concentration of NPs at $\vec{r}$ and time $t$.

3.2. Simulations: Methodology and Results. The simulation methodology employed here is based on particle tracers simulations as developed in the previous section. Now, we will have two different types of tracer particles, one corresponding to NPs and another one corresponding to colloidal particles. For simplicity, we will consider the simulation of this mixture only in the case of closed type magnetic separators. The profile of the magnetic field is shown in Figure 1. As we have seen in the previous section, this case is more easy to understand due to the near uniformity of the magnetic gradient. In this case, the magnetic gradient is pointing in the radial direction, and it has a constant magnitude. Hence, (7) gives the following expression for the radial velocity of a NP at a radial distance $r$ from the center of this cylindrical separator:

$$
v_{p}(r)=\frac{2 \mu_{0} R_{p}^{2} \rho_{p} M(H)}{9 \eta} \frac{\partial H}{\partial r},
$$


where $M(H)$ is given by (8) and (9). In the case of the colloidal particles, the radial velocity can be obtained from (12)

$$
v_{c}(r)=v_{p}(r) \frac{R_{p}}{R_{c}}\left[\frac{m_{c}}{m_{p}}-N_{f}(r, t)\right],
$$

where $N_{f}$ is the number of NPs "excluded" by the presence of a colloidal particle, and it is given by

$$
N_{f}(\vec{r}, t)=\frac{4}{3} \pi R_{c}^{3} n_{p}(\vec{r}, t) .
$$

Due to the symmetry of the problem, we consider only the radial motion of the particles so the simulations can be performed in 2 dimensions (the vertical coordinate $z$ was ignored). As in the previous section, the equation of motion of the tracer NPs, $d r / d t=v_{p}(r)$ was solved using the Verlet integration scheme. Also, we have to solve the motion of the tracer particles corresponding to the colloids. This is done as follows. At each time step (after updating the position of the NPs), we compute the concentration profile of NPs, and the function $N_{f}(r, t)$ is updated in the following way. The value of $N_{f}(r, t)$ is given by the initial value $N_{f}^{0}$ multiplied by the ratio between the NPs density found in the region comprised between $r$ and $r+\delta r$ and the initial NPs density. Different combinations of integration time step and $\delta r$ values were tested. The results reported here correspond to $\delta r=$ $L / 100$ where $L$ is the radius of the magnetic separator. Once this function is updated, the velocity of each colloidal tracer is calculated as follows. A first estimate of the velocity for each latex particle at a given time step $v_{1}\left(t_{n}\right)$ is calculated according to the NPs concentration at time $t_{n}$. Then, each latex particle is moved to a new virtual position according to this initial estimate $v_{1}\left(t_{n}\right)$. Also, we update the position for each NP at time $t_{n+1}$, the new NPs concentration is computed, and a new estimation of the velocity of each latex particle (located at their virtual positions) is calculated $v_{2}\left(t_{n+1}\right)$. Finally, the real velocity used to calculate the position at $t_{n+1}$ for each latex particle is calculated as the average of these two estimates of the latex velocities that is, $v\left(t_{n}\right)=\left(v_{1}\left(t_{n}\right)+v_{2}\left(t_{n+1}\right)\right) / 2$. This two-steps, methodology is necessary in order to account for the effect of variations in NPs concentration during the motion of the latex particles.

In this case, we have performed a single simulation for a particular case of interest which is now being realized experimentally [30]. We have considered a mixture containing a dispersion of nanoparticles identical to that considered in the previous section $(10 \mathrm{~g} / \mathrm{L}$ dispersion of superparamagnetic $\gamma$ $\mathrm{Fe}_{2} \mathrm{O}_{3}$ NPs of diameter $12 \mathrm{~nm}$ ) and colloidal particles similar to commercial latex micro spheres $(1 \mathrm{~g} / \mathrm{L}$ dispersion of colloids with diameter $900 \mathrm{~nm}$ ). In these conditions, the initial value of the quantity $N_{f}$ defined in (15) is $N_{f}^{0}=868$ in all the system. As we said before, the magnetic separator considered here is the same closed type separator with a gradient of approximately $30 \mathrm{~T} / \mathrm{m}$ discussed in Section 2.2. Under these conditions, the behavior of the NPs is the same as discussed in Section 2.2. The behavior of the latex colloids depends strongly on the value considered for $m_{c}$. The most interesting case corresponds to the case with $m_{c} / m_{p}<N_{f}$. In this situation, (14) predicts that the initial motion of the colloidal particles will be in the opposite direction to that of NPs. In our simulations, we have focused in the particular case $m_{c} / m_{p}=$ 500, which we have found realizable experimentally [30].

We have performed different simulations with a total number of $10^{6}$ tracer NPs together with $10^{4}$ tracer latex particles. The initial system configuration was prepared by placing all the particles at random positions inside the separator of radius $1.5 \mathrm{~cm}$. As in previous simulations, the solvent viscosity was set to $0.001 \mathrm{~Pa} \cdot \mathrm{s}$ which corresponds to the viscosity of water at $298 \mathrm{~K}$. The integration time step was set to $\Delta t=5 \mathrm{~s}$, and the positions of the particles were recorded at intervals of $50 \mathrm{~s}$. The simulation was performed until a simulated time of $2.9 \times 10^{5} \mathrm{~s}$, which required $131 \mathrm{~min}$ of CPU. At this point, we recall that all our calculations were performed by a $\mathrm{C}$ code developed in house, which is available under request to the authors.

Experimentally [30], it was observed that the latex particles behave in an interesting, nontrivial way. First, it was observed that latex colloids generate a sort of ring-shaped structure. Then, this ring of latex particles experiences a thinning process, and later it moves towards the walls of the system. This experimental behavior is also found in our simulations, as seen in the snapshots in Figure 6, and also it can be seen in the movies provided as supporting online information (see the movies provided in supplementary material available online at doi:10.1155/2012/678581).

The observed profiles of latex particles can be understood from the analysis of the trajectories of individual tracers. Typical trajectories for the radial distance $r(t)$ of latex particles are shown in Figure 7. Latex particles move initially towards the center of the system due to the fact that initially $m_{\text {eff }}<0$ (see (11)). As time advances, the latex motion is slown down and at certain point (different for each particle), the motion is reversed. For example, a particle starting near the center of the system $(r=0.1 \mathrm{~cm})$ reverses its motion after $1 \mathrm{~h}$, reaching the wall after a total of $31 \mathrm{~h}$. A particle starting at the wall (at $r=1.5 \mathrm{~cm}$ ) reverses its motion after $10 \mathrm{~h}$, at a distance $r=0.7 \mathrm{~cm}$ and reaches the wall $(r=1.5 \mathrm{~cm})$ also after $31 \mathrm{~h}$. This reversal of latex motion is due to the radial motion of the NPs towards the walls, which changes the local concentration of NPs. The reversal of the trajectory of a latex particle occurs when it founds a decreased local concentration of NPs at which $m_{\text {eff }}=0((12)$ gives a threshold NP concentration of $5.8 \mathrm{~g} / \mathrm{L}$ for our case). This initial motion towards the center of the system generates the observed formation and thinning of the ring profile of latex particles shown in Figure 6. The subsequent motion of the ring profile of latex towards the wall corresponds to the time at which all latex particles have inverted their motion. All this process is also illustrated in the accompanying movies, showing the motion of latex particles superimposed to the evolution of the concentration profiles of NPs.

3.3. Simplified Model. Although our simulations described in the previous section are not particularly costly from the computational point of view, they require the use of relatively large amounts of disk space to store the tracer trajectories and later analysis to obtain relevant quantities such as concentration profiles and number of particles remaining inside the magnetic separator. For this reason, it could be convenient to 


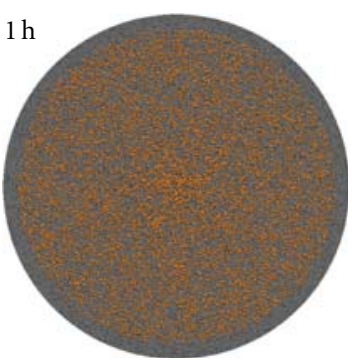

(a)

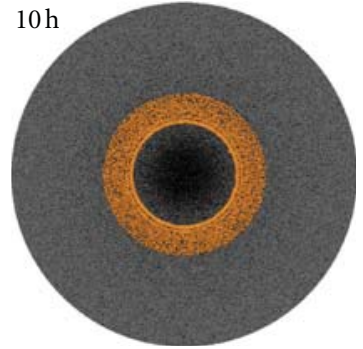

(b)

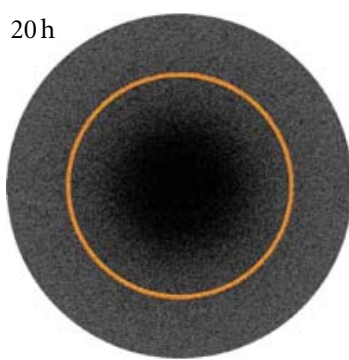

(c)

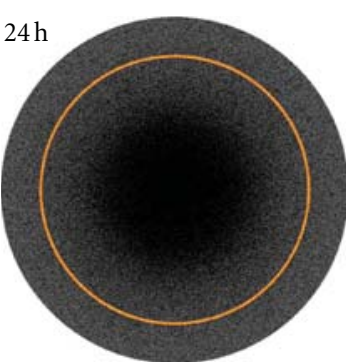

(d)

FIGURE 6: Snapshots from simulations of the magnetophoresis of an aqueous dispersion of $\gamma$ - $\mathrm{Fe}_{2} \mathrm{O}_{3}$ superparamagnetic nanoparticles (grey) and latex polystyrene particles (orange) under a magnetic gradient of $30 \mathrm{~T} / \mathrm{m}$ at different times $(1 \mathrm{~h}, 10 \mathrm{~h}, 20 \mathrm{~h}$, and $24 \mathrm{~h}$ resp.).

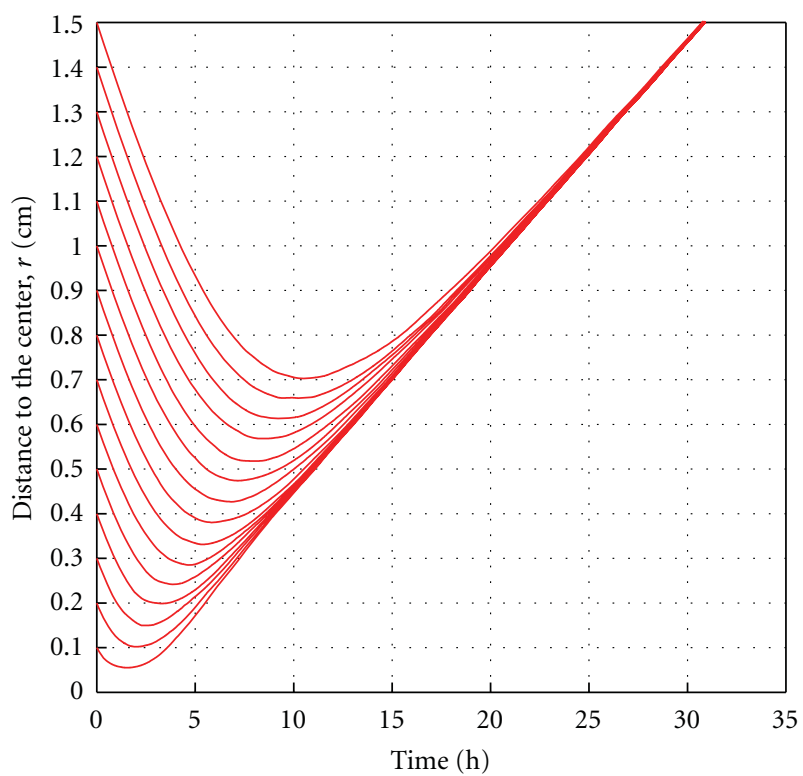

Figure 7: Examples of 15 trajectories obtained in simulations corresponding to tracer latex particles immersed in a dispersion of NPs starting from different distances to the center of the system (see details in the text.)

develop a simplified approach amenable of solution without the need of performing computer simulations.

The motion of the latex particles observed in the simulations can be described with reasonable accuracy with a simple equation. The basic idea is to disregard the radial dependence in the magnetophoretic velocity of the NPs in (13) and assume that the NPs move at constant velocity $v_{p}^{s}$ which is the magnetophoretic velocity at magnetic saturation

$$
v_{p}^{s}=\frac{2 R_{p}^{2}}{9 \eta} \mu_{0}\left(\frac{\partial H}{\partial r}\right) M_{s} \rho_{p}
$$

This approximation is justified by the observation that the magnetic field observed in most parts of the magnetic separator (see Figure 1) is large enough to saturate the NPs. In the case of the $10 \mathrm{~g} / \mathrm{L}$ suspension of $\gamma-\mathrm{Fe}_{2} \mathrm{O}_{3} \mathrm{NPs}$ under $30 \mathrm{~T} / \mathrm{m}$ considered in our previous subsection, we have $v_{p}^{s}=$
$7.9 \times 10^{-8} \mathrm{~m} / \mathrm{s}$. Within this approximation, the concentration profile of NPs is given approximately by

$$
\begin{gathered}
n_{p}(r, t)=n_{0}\left(1-\frac{v_{p}^{s} t}{r}\right) \quad \text { for } r>v_{p} t \\
n_{p}(r, t)=0 \quad \text { for } r<v_{p} t .
\end{gathered}
$$

Using (16)-(18) in (14), we obtain that the trajectory of a latex particle obeys the differential equation

$$
\frac{d r}{d t}=v_{c}(r)=v_{p}^{s} \frac{R_{p}}{R_{c}}\left[\frac{m_{c}}{m_{p}}-\frac{4}{3} \pi R_{c}^{3} n_{0}\left(1-\frac{v_{p}^{s} t}{r}\right)\right] .
$$

Equation (19) is a first-order differential equation which can be solved numerically to obtain the trajectory $r(t)$ for a latex particle initially at a position $r(t=0)=r_{0}$. In Figure 8 we compare the predictions of (19) with the results obtained from tracer simulations. In general, numerical solutions of (19) give a reasonable approximation to colloid trajectories with differences of the order of $10 \%$ with simulations. This result is remarkable in view of the apparently strong approximations involved in their derivation (see (16) and (18)). Therefore, in order to estimate systematically the effect of the different parameters of the system (e.g., the effect of the value of $m_{c}$ ), it could be convenient in practice to solve numerically (19) instead of performing a full simulation. However, for more complex magnetic field geometries, to get analytical solutions becomes much more difficult, and numerical simulations as the ones presented here would be necessary.

\section{Conclusions}

In this work, we have presented a low-cost simulation strategy based on the concept of particle tracers aimed to tackle the magnetophoresis process in the noncooperative magnetophoretic regime. We have successfully validated this simulation approach by comparing the results obtained against existing experimental and also analytical results obtained for the separation process of a colloidal dispersion of $\gamma-\mathrm{Fe}_{2} \mathrm{O}_{3}$ superparamagnetic nanoparticles in an aqueous solution. Thanks to this methodology, we have been able to evaluate different key factors involved in the magnetophoretic 


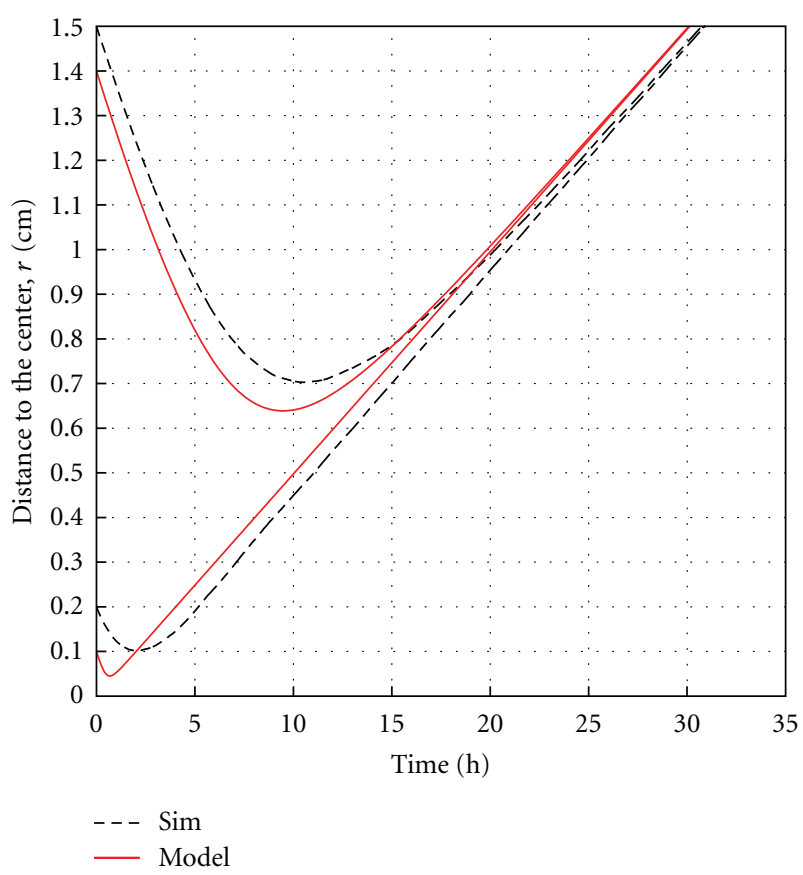

Figure 8: Comparison of trajectories of two tracer latex particles as obtained in the simulations and by numerical solution of (19).

separation process. Regarding the separator design, we have shown that the homogeneous magnetophoretic conditions created by a closed type separator (high magnetic field over almost the whole sample and constant magnetic gradient) enhance the separation process, providing more control over the process and reducing the expected separation time when compared to the open-type version of the separator. We have also extended that methodology to solutions of colloidal particles in aqueous solutions of superparamagnetic nanoparticles in the closed type geometry. The simulation performed in this case is able to account for the ringlike structure expected in some experimental situations and agrees with the simplified numerical model proposed.

\section{Acknowledgments}

This work was supported by the Spanish Government Grants no. FIS2009-13370-C02-02, PET2008-02-81-01/02, and CONSOLIDER-NANOSELECT-CSD2007-00041. J. Faraudo and J. Camacho are also supported by the Catalan Government Grant no. 2009SGR164. The authors thank LL. M. Martínez from SEPMAG Technologies for help regarding the calculation of the profiles of the magnetic fields and for extensive discussions.

\section{References}

[1] C. T. Yavuz, A. Prakash, J. T. Mayo, and V. L. Colvin, "Magnetic separations: from steel plants to biotechnology," Chemical Engineering Science, vol. 64, no. 10, pp. 2510-2521, 2009.
[2] C. De Latour, "Magnetic separation in water pollution control," IEEE Transactions on Magnetics, vol. 9, no. 3, pp. 314316, 1973.

[3] G. Mariani, M. Fabbri, F. Negrini, and P. L. Ribani, "HighGradient Magnetic Separation of pollutant from wastewaters using permanent magnets," Separation and Purification Technology, vol. 72, no. 2, pp. 147-155, 2010.

[4] C. T. Yavuz, J. T. Mayo, W. W. Yu et al., "Low-field magnetic separation of monodisperse $\mathrm{Fe}_{3} \mathrm{O}_{4}$ nanocrystals," Science, vol. 314, no. 5801, pp. 964-967, 2006.

[5] J. L. Corchero and A. Villaverde, "Biomedical applications of distally controlled magnetic nanoparticles," Trends in Biotechnology, vol. 27, no. 8, pp. 468-476, 2009.

[6] K. M. Krishnan, "Biomedical nanomagnetics: a spin through possibilities in imaging, diagnostics, and therapy," IEEE Transactions on Magnetics, vol. 46, no. 7, Article ID 5439840, pp. 2523-2558, 2010.

[7] I. Safarik and M. Safarikova, "Magnetic techniques for the isolation and purification of proteins and peptides," BioMagnetic Research and Technology, vol. 2, no. 1, article no. 7, 2004.

[8] G. Friedman and B. Yellen, "Magnetic separation, manipulation and assembly of solid phase in fluids," Current Opinion in Colloid and Interface Science, vol. 10, no. 3-4, pp. 158-166, 2005.

[9] G. D. Moeser, K. A. Roach, W. H. Green, T. A. Hatton, and P. E. Laibinis, "High-gradient magnetic separation of coated magnetic nanoparticles," AIChE Journal, vol. 50, no. 11, pp. 2835-2848, 2004.

[10] C. P. Bean and J. D. Livingston, "Superparamagnetism," Journal of Applied Physics, vol. 30, no. 4, pp. S120-S129, 1959.

[11] D. Leun and A. K. Sengupta, "Preparation and characterization of magnetically active polymeric particles (MAPPs) for complex environmental separations," Environmental Science and Technology, vol. 34, no. 15, pp. 3276-3282, 2000.

[12] E. Taboada, R. Solanas, E. Rodríguez, R. Weissleder, and A. Roig, "Supercritical-fluid-assisted one-pot synthesis of biocompatible core $\left(\gamma-\mathrm{Fe}_{2} \mathrm{O}_{3}\right) / \operatorname{shell}\left(\mathrm{SiO}_{2}\right)$ nanoparticles as high relaxivity $\mathrm{T}_{2}$-contrast agents for magnetic resonance imaging," Advanced Functional Materials, vol. 19, no. 14, pp. 2319-2324, 2009.

[13] G. De Las Cuevas, J. Faraudo, and J. Camacho, "Low-gradient magnetophoresis through field-induced reversible aggregation," Journal of Physical Chemistry C, vol. 112, no. 4, pp. 945-950, 2008.

[14] J. Faraudo and J. Camacho, "Cooperative magnetophoresis of superparamagnetic colloids: theoretical aspects," Colloid and Polymer Science, vol. 288, no. 2, pp. 207-215, 2010.

[15] M. Benelmekki, C. Caparros, A. Montras, R. Gonçalves, S. Lanceros-Mendez, and L. M. Martinez, "Horizontal low gradient magnetophoresis behaviour of iron oxide nanoclusters at the different steps of the synthesis route," Journal of Nanoparticle Research, vol. 13, no. 8, pp. 3199-3206, 2011.

[16] M. Benelmekki, A. Montras, A. J. Martins, P. J.G. Coutinho, and L. M. Martinez, "Magnetophoresis behaviour at low gradient magnetic field and size control of nickel single core nanobeads," Journal of Magnetism and Magnetic Materials, vol. 323, no. 15, pp. 1945-1949, 2011.

[17] J. S. Andreu, J. Camacho, J. Faraudo, M. Benelmekki, C. Rebollo, and L. M. Martínez, "Simple analytical model for the magnetophoretic separation of superparamagnetic dispersions in a uniform magnetic gradient," Physical Review E, vol. 84, no. 2, Article ID 021402, 2011.

[18] G. P. Hatch and R. E. Stelter, "Magnetic design considerations for devices and particles used for biological high-gradient 
magnetic separation (HGMS) systems," Journal of Magnetism and Magnetic Materials, vol. 225, no. 1-2, pp. 262-276, 2001.

[19] S. J. Hershberger, A. Parakka, B. Trudeau, C. Patel, and P. Schultz, "Scalable magnetic designs to achieve comparable capture rates and capture efficiency across multiple vessel diameters," AIP Conference Proceedings, vol. 1311, pp. 351-362, 2010.

[20] J. S. Andreu, J. Camacho, J. Faraudo, M. Benelmekki, C. Rebollo, and L. Martínez, "Magnetophoretic separation of superparamagnetic dispersions in a uniform magnetic gradient: experimental study and analytical solution," in Proceedings of the 4th Iberian Meeting on Colloids and Interfaces, pp. 191-197, 2011.

[21] R. Kaiser and G. Miskolczy, "Magnetic properties of stable dispersions of subdomain magnetite particles," Journal of Applied Physics, vol. 41, no. 3, pp. 1064-1072, 1970.

[22] R. Rosensweig, Ferrohydrodynamics, Cambridge University Press, New York, NY, USA, 1st edition, 1985.

[23] D. X. Chen, A. Sanchez, E. Taboada, A. Roig, N. Sun, and H. C. $\mathrm{Gu}$, "Size determination of superparamagnetic nanoparticles from magnetization curve," Journal of Applied Physics, vol. 105, no. 8, Article ID 083924, 2009.

[24] J. J. Monaghan, "Particle methods for hydrodynamics," Computer Physics Reports, vol. 3, no. 2, pp. 71-124, 1985.

[25] D. Frenkel and B. Smit, Understanding Molecular Simulations, Academic Press, 2nd edition, 2001.

[26] B. B. Yellen, O. Hovorka, and G. Friedman, "Arranging matter by magnetic nanoparticle assemblers," Proceedings of the National Academy of Sciences of the United States of America, vol. 102, no. 25, pp. 8860-8864, 2005.

[27] R. M. Erb, H. S. Son, B. Samanta, V. M. Rotello, and B. B. Yellen, "Magnetic assembly of colloidal superstructures with multipole symmetry," Nature, vol. 457, no. 7232, pp. 9991002, 2009.

[28] R. M. Erb and B. B. Yellen, "Concentration gradients in mixed magnetic and nonmagnetic colloidal suspensions," Journal of Applied Physics, vol. 103, no. 7, Article ID 07A312, 2008.

[29] R. M. Erb, D. S. Sebba, A. A. Lazarides, and B. B. Yellen, "Magnetic field induced concentration gradients in magnetic nanoparticle suspensions: theory and experiment," Journal of Applied Physics, vol. 103, no. 6, Article ID 063916, 2008.

[30] M. Benelmekki, Ll. M Martínez, J. Andreu, J. Faraudo, and J. Camacho, "Magnetophoresis of colloidal particles in a dispersion of superparamagnetic nanoparticles: theory and experiments," Soft Matter. In press. 

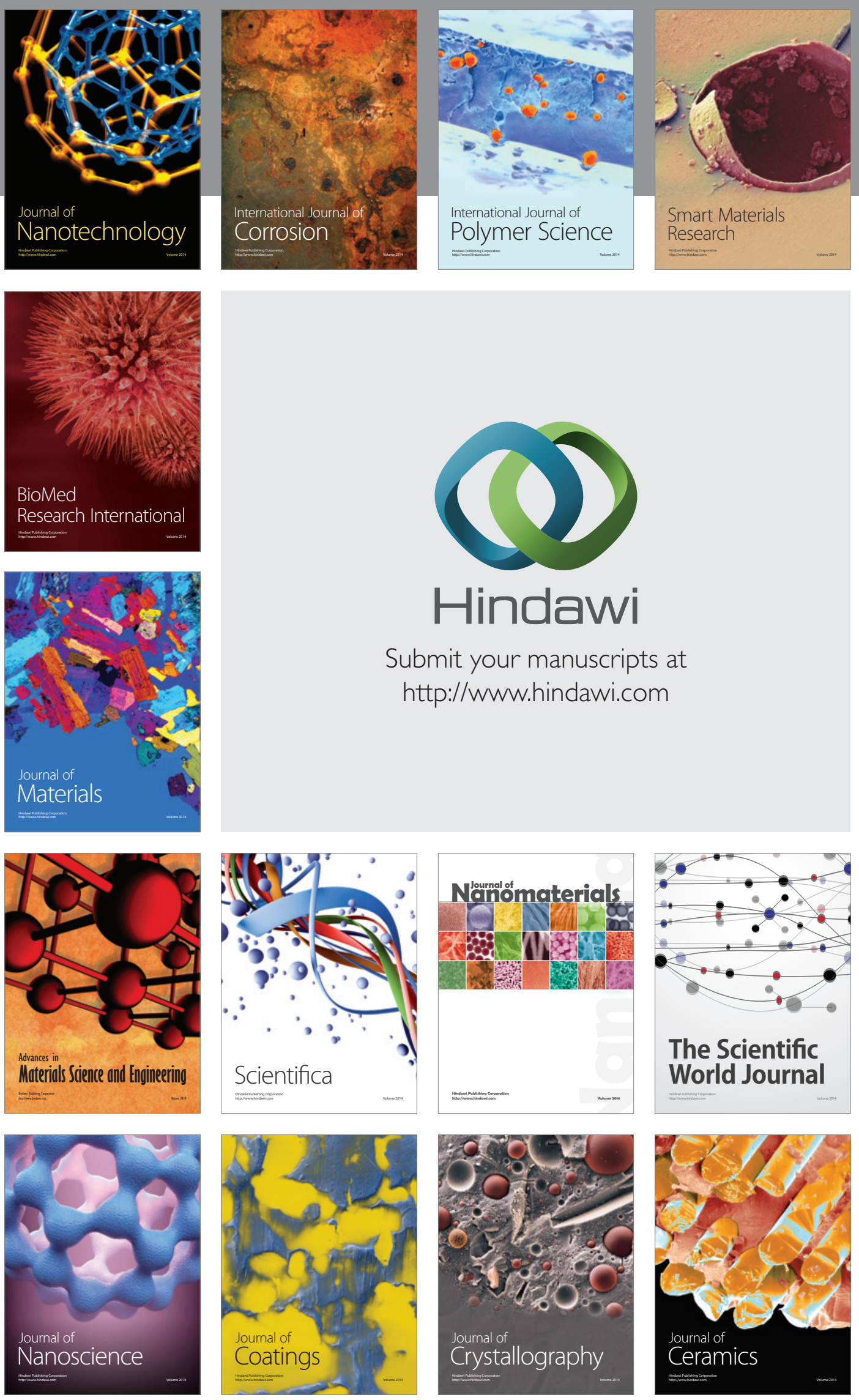

The Scientific World Journal

Submit your manuscripts at

http://www.hindawi.com

\section{World Journal}

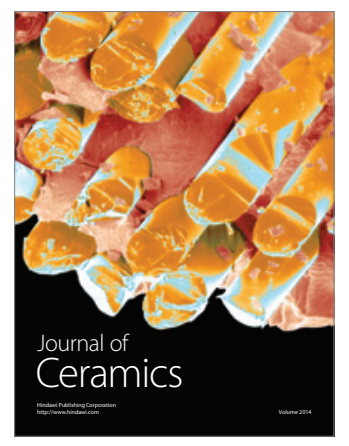

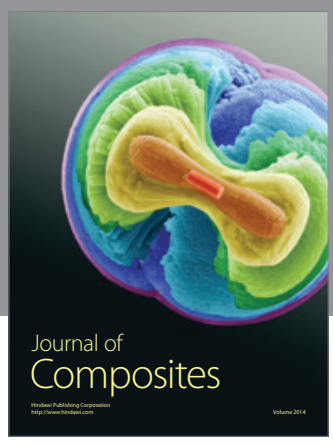
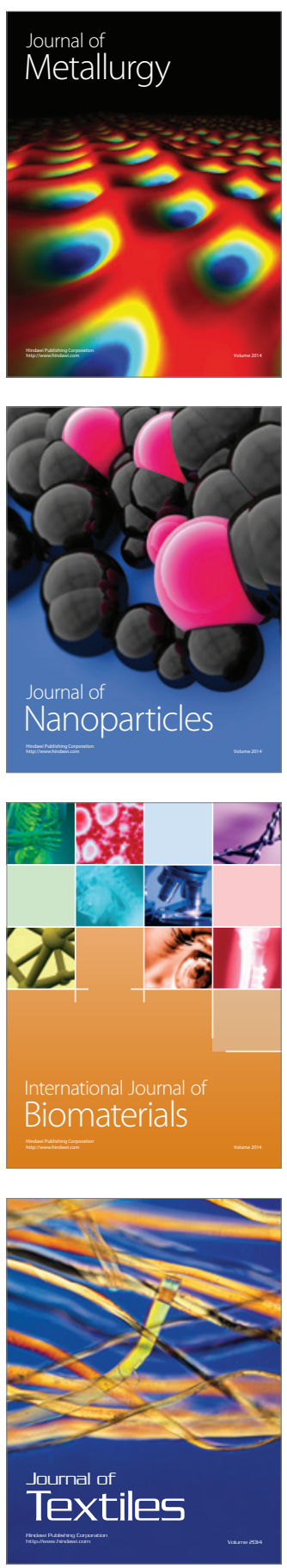